\title{
On the spectral norms of $r$-circulant matrices with the biperiodic Fibonacci and Lucas numbers
}

\section{Cahit Köme and Yasin Yazlik *i)}

\section{"Correspondence:}

yyazlik@nevsehir.edu.tr

Department of Mathematics,

Nevşehir Hacı Bektaş Veli University,

Nevşehir, 50300, Turkey

\begin{abstract}
In this paper, we present new upper and lower bounds for the spectral norms of the $r$-circulant matrices $Q=C_{r}\left(\left(\frac{b}{a}\right)^{\frac{\xi(1)}{2}} q_{0},\left(\frac{b}{a}\right)^{\frac{\xi(2)}{2}} q_{1},\left(\frac{b}{a}\right)^{\frac{\xi(3)}{2}} q_{2}, \ldots,\left(\frac{b}{a}\right)^{\frac{\xi(n)}{2}} q_{n-1}\right)$ and $L=C_{r}\left(\left(\frac{b}{a}\right)^{\frac{\xi(0)}{2}} I_{0}\left(\frac{b}{a}\right)^{\frac{\xi(1)}{2}} I_{1},\left(\frac{b}{a}\right)^{\frac{\xi(2)}{2}} I_{2}, \ldots,\left(\frac{b}{a}\right)^{\frac{\xi(n-1)}{2}} I_{n-1}\right)$ whose entries are the biperiodic Fibonacci and biperiodic Lucas numbers, respectively. Finally, we obtain lower and upper bounds for the spectral norms of Kronecker and Hadamard products of $Q$ and $L$ matrices.
\end{abstract}

Keywords: biperiodic Fibonacci number; biperiodic Lucas number; r-circulant matrix; norm

\section{Introduction}

For $n \in \mathbb{N}_{0}$, the Fibonacci and Lucas numbers are defined by $F_{n+2}=F_{n+1}+F_{n}$ and $L_{n+2}=$ $L_{n+1}+L_{n}$ with the initial conditions $F_{0}=0, F_{1}=1$ and $L_{0}=2, L_{1}=1$, respectively. In recent years, there are several applications and generalizations of Fibonacci and Lucas numbers [1-12]. For example, Falcon and Plaza introduced the $k$-Fibonacci sequence by studying the recursive application of two geometrical transformations used in the well-known 4triangle longest-edge (4TLE) partition [6]. Edson and Yayenie [3] presented a new generalization of the Fibonacci sequence: for $n \in \mathbb{N}_{0}$,

$$
q_{0}=0, \quad q_{1}=1, \quad q_{n+2}= \begin{cases}a q_{n+1}+q_{n} & \text { if } n \text { is even, } \\ b q_{n+1}+q_{n} & \text { if } n \text { is odd }\end{cases}
$$

They also obtained an extended Binet formula for this sequence:

$$
q_{n}=\left(\frac{a^{1-\xi(n)}}{a b^{\left\lfloor\frac{n}{2}\right\rfloor}}\right) \frac{\alpha^{n}-\beta^{n}}{\alpha-\beta}, \quad n \in \mathbb{N}_{0} .
$$

Afterward, Bilgici [4] defined generalized the Lucas sequence by the following recurrence relation: for $n \in \mathbb{N}_{0}$,

$$
l_{0}=2, \quad l_{1}=a, \quad l_{n+2}= \begin{cases}b l_{n+1}+l_{n} & \text { if } n \text { is even, } \\ a l_{n+1}+l_{n} & \text { if } n \text { is odd }\end{cases}
$$

( T) The Author(s) 2017. This article is distributed under the terms of the Creative Commons Attribution 4.0 International License (http://creativecommons.org/licenses/by/4.0/), which permits unrestricted use, distribution, and reproduction in any medium, provided you give appropriate credit to the original author(s) and the source, provide a link to the Creative Commons license, and indicate if changes were made. 
and The Binet formula for this sequence is

$$
l_{n}=\left(\frac{a^{\xi(n)}}{a b^{\left\lfloor\frac{n+1}{2}\right\rfloor}}\right)\left(\alpha^{n}+\beta^{n}\right), \quad n \in \mathbb{N}_{0} .
$$

In Eqs. (2) and (4), $\alpha=\frac{a b+\sqrt{a^{2} b^{2}+4 a b}}{2}$ and $\beta=\frac{a b-\sqrt{a^{2} b^{2}+4 a b}}{2}$ are the roots of the characteristic equation of $x^{2}-a b x-a b=0$, and $\xi(n)=n-2\left\lfloor\frac{n}{2}\right\rfloor$.

In recent years, there have been several studies on the norms, determinants, and inverses of circulant and $r$-circulant matrices whose entries are special integer sequences [13-27]. For example, Shen and Cen [18] found upper and lower bounds for the spectral norms of $r$-circulant matrices in the forms $A=C_{r}\left(F_{0}, F_{1}, F_{2}, \ldots, F_{n-1}\right)$ and $B=C_{r}\left(L_{0}, L_{1}, L_{2}, \ldots, L_{n-1}\right)$. They also obtained some bounds for the spectral norms of Kronecker and Hadamard products of $A$ and $B$. Afterward, Shen and Cen [19] gave the upper and lower bounds for the spectral norms of the matrices $A=C_{r}\left(F_{k, 0}, F_{k, 1}, F_{k, 2}, \ldots, F_{k, n-1}\right)$ and $B=C_{r}\left(L_{k, 0}, L_{k, 1}, L_{k, 2}, \ldots, L_{k, n-1}\right)$. They also presented some bounds for the spectral norms of Hadamard and Kronecker products of these matrices. Bahşi [16] studied the norms of $r$-circulant matrices $H_{r}=\operatorname{Circr}\left(H_{0}^{(k)}, H_{1}^{(k)}, H_{2}^{(k)}, \ldots, H_{n-1}^{(k)}\right)$ and $\widehat{H_{r}}=$ $\operatorname{Circr}\left(H_{k}^{(0)}, H_{k}^{(1)}, H_{k}^{(2)}, \ldots, H_{k}^{(n-1)}\right)$, where $H_{n}^{(k)}$ denotes the $n$th hyperharmonic number of order $r$.

Inspired by these studies, in this paper, we compute spectral norms of $r$-circulant matrices whose entries are the biperiodic Fibonacci and biperiodic Lucas numbers. This study consists of three sections. The first one is the introduction. In the second section, we give some new theorems, corollaries, and some important results. We give a concise conclusion in the last section.

Definition 1.1 For any given $c_{0}, c_{1}, c_{2}, \ldots, c_{n-1} \in \mathbb{C}$, the $r$-circulant matrix $C_{r}=\left(c_{i j}\right)_{n \times n}$ is defined by

$$
C_{r}=\left[\begin{array}{cccccc}
c_{0} & c_{1} & c_{2} & \ldots & c_{n-2} & c_{n-1} \\
r c_{n-1} & c_{0} & c_{1} & \ldots & c_{n-3} & c_{n-2} \\
r c_{n-2} & r c_{n-1} & c_{0} & \ldots & c_{n-4} & c_{n-3} \\
\vdots & \vdots & \vdots & \ddots & \vdots & \vdots \\
r c_{2} & r c_{3} & r c_{4} & \ldots & c_{0} & c_{1} \\
r c_{1} & r c_{2} & r c_{3} & \ldots & r c_{n-1} & c_{0}
\end{array}\right] .
$$

It is clear that, for $r=1, C_{r}$ turns into a classical circulant matrix. Let us take any $A=\left[a_{i j}\right] \in$ $M_{n, n}(\mathbb{C})$. The Frobenius norm of the matrix $A$ is defined by

$$
\|A\|_{F}=\left[\sum_{i=1}^{m} \sum_{j=1}^{n}\left|a_{i j}\right|^{2}\right]^{\frac{1}{2}}
$$

Also, the spectral norm of the matrix $A$ is given by

$$
\|A\|_{2}=\sqrt{\max _{1 \leq i \leq n} \lambda_{i}\left(A^{H} A\right),}
$$


where $\lambda_{i}\left(A^{H} A\right)$ are the eigenvalues of $A^{H} A$ such that $A^{H}$ is the conjugate transpose of $A$. Then, the well-known inequality [28] is given by

$$
\frac{1}{\sqrt{n}}\|A\|_{F} \leq\|A\|_{2} \leq\|A\|_{F}
$$

Lemma 1.2 ([28]) For any matrices $A, B \in M_{m, n}(\mathbb{C})$, we have

$$
\|A \circ B\|_{2} \leq\|A\|_{2}\|B\|_{2}
$$

where $A \circ B$ is the Hadamard product of $A$ and $B$.

Lemma 1.3 ([28]) For any matrices $A \in M_{m, n}(\mathbb{C})$ and $B \in M_{p, q}(\mathbb{C})$, we have

$$
\|A \otimes B\|_{2}=\|A\|_{2}\|B\|_{2}
$$

where $A \otimes B$ is the Kronecker product of $A$ and $B$.

Lemma 1.4 ([29]) For any matrices $A=\left[a_{i j}\right] \in M_{n, n}(\mathbb{C})$ and $B=\left[b_{i j}\right] \in M_{n, n}(\mathbb{C})$, we have

$$
\|A \circ B\|_{2} \leq r_{1}(A) c_{1}(B)
$$

where $A \circ B$ is the Hadamard product, $r_{1}(A)=\max _{1 \leq i \leq n} \sqrt{\sum_{j=1}^{n}\left|a_{i j}\right|^{2}}$, and $c_{1}(B)=$ $\max _{1 \leq j \leq n} \sqrt{\sum_{i=1}^{n}\left|b_{i j}\right|^{2}}$.

Theorem 1.5 ([5]) For any positive integer $n$, we have

$$
\sum_{k=1}^{n}\left(\frac{b}{a}\right)^{\xi(k+1)} q_{k}^{2}=\left(\frac{1}{a}\right) q_{n} q_{n+1}
$$

\section{Main results}

In this section, we first give the sum of squares of biperiodic Lucas numbers.

Theorem 2.1 For any positive integer $m$, we have

$$
\sum_{k=1}^{m}\left(\frac{b}{a}\right)^{\xi(k)} l_{k}^{2}=\left(\frac{1}{a}\right) l_{m+1} l_{m}-2
$$

Proof Using the Binet formula of the biperiodic Lucas numbers, we have

$$
\begin{cases}l_{k}^{2}=\left(\frac{\alpha^{2}}{a b}\right)^{k}+\left(\frac{\beta^{2}}{a b}\right)^{k}+2(-1)^{k} & \text { if } k \text { is even, } \\ l_{k}^{2}=\left(\frac{a}{b}\right)\left[\left(\frac{\alpha^{2}}{a b}\right)^{k}+\left(\frac{\beta^{2}}{a b}\right)^{k}+2(-1)^{k}\right] & \text { if } k \text { is odd. }\end{cases}
$$

Therefore, for any $k \geq 1$,

$$
\left(\frac{b}{a}\right)^{\xi(k)} l_{k}^{2}=\left(\frac{\alpha^{2}}{a b}\right)^{k}+\left(\frac{\beta^{2}}{a b}\right)^{k}+2(-1)^{k} .
$$


Using the properties $a b(\alpha+1)=\alpha^{2}$ and $a b(\beta+1)=\beta^{2}$, we get

$$
\begin{aligned}
\sum_{k=1}^{m}\left(\frac{b}{a}\right)^{\xi(k)} l_{k}^{2} & =\sum_{k=1}^{m}\left(\frac{\alpha^{2}}{a b}\right)^{k}+\sum_{k=1}^{m}\left(\frac{\beta^{2}}{a b}\right)^{k}+\sum_{k=1}^{m} 2(-1)^{k} \\
& =\frac{\left(\frac{\alpha^{2}}{a b}\right)^{m+1}-\left(\frac{\alpha^{2}}{a b}\right)}{\left(\frac{\alpha^{2}}{a b}\right)-1}+\frac{\left(\frac{\beta^{2}}{a b}\right)^{m+1}-\left(\frac{\beta^{2}}{a b}\right)}{\left(\frac{\beta^{2}}{a b}\right)-1}+(-1)^{m}-1 \\
& =\frac{1}{(a b)^{m+1}}\left[\alpha^{2 m+1}+\beta^{2 m+1}-(-1)^{m}\right]-2 .
\end{aligned}
$$

Observe that

$$
\left(\frac{1}{a}\right) l_{m} l_{m+1}=\frac{1}{(a b)^{m+1}}\left[\alpha^{2 m+1}+\beta^{2 m+1}-(-1)^{m}\right] .
$$

Therefore,

$$
\sum_{k=1}^{m}\left(\frac{b}{a}\right)^{\xi(k)} l_{k}^{2}=\left(\frac{1}{a}\right) l_{m+1} l_{m}-2
$$

Theorem 2.2 Let $Q=C_{r}\left(\left(\frac{b}{a}\right)^{\frac{\xi(1)}{2}} q_{0},\left(\frac{b}{a}\right)^{\frac{\xi(2)}{2}} q_{1},\left(\frac{b}{a}\right)^{\frac{\xi(3)}{2}} q_{2}, \ldots,\left(\frac{b}{a}\right)^{\frac{\xi(n)}{2}} q_{n-1}\right)$ be an r-circulant matrix. Then, for $r \in \mathbb{C}$, we have:

if $|r| \geq 1$, then

$$
\sqrt{\frac{q_{n} q_{n-1}}{a}} \leq\|Q\|_{2} \leq|r| \frac{q_{n} q_{n-1}}{a}
$$

if $|r|<1$, then

$$
|r| \sqrt{\frac{q_{n} q_{n-1}}{a}} \leq\|Q\|_{2} \leq \sqrt{(n-1) \frac{q_{n} q_{n-1}}{a}} .
$$

Proof The matrix $Q$ is of the form

$$
Q=\left[\begin{array}{ccccc}
\left(\frac{b}{a}\right)^{\frac{\xi(1)}{2}} q_{0} & \left(\frac{b}{a}\right)^{\frac{\xi(2)}{2}} q_{1} & \left(\frac{b}{a}\right)^{\frac{\xi(3)}{2}} q_{2} & \ldots & \left(\frac{b}{a}\right)^{\frac{\xi(n)}{2}} q_{n-1} \\
r\left(\frac{b}{a}\right)^{\frac{\xi(n)}{2}} q_{n-1} & \left(\frac{b}{a}\right)^{\frac{\xi(1)}{2}} q_{0} & \left(\frac{b}{a}\right)^{\frac{\xi(2)}{2}} q_{1} & \ldots & \left(\frac{b}{a}\right)^{\frac{\xi(n-1)}{2}} q_{n-2} \\
r\left(\frac{b}{a}\right)^{\frac{\xi(n-1)}{2}} q_{n-2} & r\left(\frac{b}{a}\right)^{\frac{\xi(n)}{2}} q_{n-1} & \left(\frac{b}{a}\right)^{\frac{\xi}{2}} q_{0} & \ldots & \left(\frac{b}{a}\right)^{\frac{\xi(n-2)}{2}} q_{n-3} \\
\vdots & \vdots & \vdots & \ddots & \vdots \\
r\left(\frac{b}{a}\right)^{\frac{\xi(2)}{2}} q_{1} & r\left(\frac{b}{a}\right)^{\frac{\xi(3)}{2}} q_{2} & r\left(\frac{b}{a}\right)^{\frac{\xi(4)}{2}} q_{3} & \ldots & \left(\frac{b}{a}\right)^{\frac{\xi(1)}{2}} q_{0}
\end{array}\right] .
$$

Then we have

$$
\|Q\|_{F}^{2}=\sum_{k=0}^{n-1}(n-k)\left(\frac{b}{a}\right)^{\xi(k+1)} q_{k}^{2}+\sum_{k=1}^{n-1} k|r|^{2}\left(\frac{b}{a}\right)^{\xi(k+1)} q_{k}^{2} .
$$


Hence, for $|r| \geq 1$, using Eq. (8), we obtain

$$
\begin{aligned}
\|Q\|_{F}^{2} & \geq \sum_{k=0}^{n-1}(n-k)\left(\frac{b}{a}\right)^{\xi(k+1)} q_{k}^{2}+\sum_{k=1}^{n-1} k\left(\frac{b}{a}\right)^{\xi(k+1)} q_{k}^{2} \\
& =n \sum_{k=0}^{n-1}\left(\frac{b}{a}\right)^{\xi(k+1)} q_{k}^{2} \\
& =n\left(\frac{q_{n} q_{n-1}}{a}\right),
\end{aligned}
$$

that is,

$$
\frac{1}{\sqrt{n}}\|Q\|_{F} \geq \sqrt{\frac{q_{n} q_{n-1}}{a}} .
$$

From (6) we have

$$
\|Q\|_{2} \geq \sqrt{\frac{q_{n} q_{n-1}}{a}} .
$$

Now, for $|r| \geq 1$, we give an bound for the spectral norm of the matrix $Q$. Let the matrices $B$ and $C$ be

$$
B=\left[\begin{array}{ccccc}
r\left(\frac{b}{a}\right)^{\frac{\xi(1)}{2}} q_{0} & 1 & 1 & \ldots & 1 \\
r\left(\frac{b}{a}\right)^{\frac{\xi(n)}{2}} q_{n-1} & r\left(\frac{b}{a}\right)^{\frac{\xi(1)}{2}} q_{0} & 1 & \ldots & 1 \\
r\left(\frac{b}{a}\right)^{\frac{\xi(n-1)}{2}} q_{n-2} & r\left(\frac{b}{a}\right)^{\frac{\xi(n)}{2}} q_{n-1} & r\left(\frac{b}{a}\right)^{\frac{\xi(1)}{2}} q_{0} & \ldots & 1 \\
\vdots & \vdots & \vdots & \ddots & \vdots \\
r\left(\frac{b}{a}\right)^{\frac{\xi(2)}{2}} q_{1} & r\left(\frac{b}{a}\right)^{\frac{\xi(3)}{2}} q_{2} & r\left(\frac{b}{a}\right)^{\frac{\xi(4)}{2}} q_{3} & \ldots & r\left(\frac{b}{a}\right)^{\frac{\xi(1)}{2}} q_{0}
\end{array}\right]
$$

and

$$
C=\left[\begin{array}{ccccc}
\left(\frac{b}{a}\right)^{\frac{\xi(1)}{2}} q_{0} & \left(\frac{b}{a}\right)^{\frac{\xi(2)}{2}} q_{1} & \left(\frac{b}{a}\right)^{\frac{\xi(3)}{2}} q_{2} & \ldots & \left(\frac{b}{a}\right)^{\frac{\xi(n)}{2}} q_{n-1} \\
1 & \left(\frac{b}{a}\right)^{\frac{\xi(1)}{2}} q_{0} & \left(\frac{b}{a}\right)^{\frac{\xi(2)}{2}} q_{1} & \ldots & \left(\frac{b}{a}\right)^{\frac{\xi(n-1)}{2}} q_{n-2} \\
1 & 1 & \left(\frac{b}{a}\right)^{\frac{\xi(1)}{2}} q_{0} & \ldots & \left(\frac{b}{a}\right)^{\frac{\xi(n-2)}{2}} q_{n-3} \\
\vdots & \vdots & \vdots & \ddots & \vdots \\
1 & 1 & 1 & \ldots & \left(\frac{b}{a}\right)^{\frac{\xi(1)}{2}} q_{0}
\end{array}\right],
$$

so that $Q=B \circ C$. Then we obtain

$$
\begin{aligned}
& r_{1}(B)=\max _{1 \leq i \leq n} \sqrt{\sum_{j=1}^{n}\left|b_{i j}\right|^{2}}=\sqrt{|r|^{2} \sum_{k=0}^{n-1}\left(\frac{b}{a}\right)^{\xi(k+1)} q_{k}^{2}}=|r| \sqrt{\frac{q_{n} q_{n-1}}{a}} \\
& c_{1}(C)=\max _{1 \leq j \leq n} \sqrt{\sum_{i=1}^{n}\left|c_{i j}\right|^{2}}=\sqrt{\sum_{k=0}^{n-1}\left(\frac{b}{a}\right)^{\xi(k+1)} q_{k}^{2}}=\sqrt{\frac{q_{n} q_{n-1}}{a}}
\end{aligned}
$$

By Lemma 1.4 we have

$$
\|Q\|_{2} \leq r_{1}(B) c_{1}(C)=|r| \frac{q_{n} q_{n-1}}{a} .
$$


Thus,

$$
\sqrt{\frac{q_{n} q_{n-1}}{a}} \leq\|Q\|_{2} \leq|r| \frac{q_{n} q_{n-1}}{a} .
$$

On the other hand, for $|r|<1$, we have

$$
\begin{aligned}
\|Q\|_{F}^{2} & \geq \sum_{k=0}^{n-1}(n-k)|r|^{2}\left(\frac{b}{a}\right)^{\xi(k+1)} q_{k}^{2}+\sum_{k=1}^{n-1} k|r|^{2}\left(\frac{b}{a}\right)^{\xi(k+1)} q_{k}^{2} \\
& =n|r|^{2} \sum_{k=0}^{n-1}\left(\frac{b}{a}\right)^{\xi(k+1)} q_{k}^{2} \\
& =n|r|^{2}\left(\frac{q_{n} q_{n-1}}{a}\right)
\end{aligned}
$$

that is,

$$
\frac{1}{\sqrt{n}}\|Q\|_{F} \geq|r| \sqrt{\frac{q_{n} q_{n-1}}{a}} .
$$

Thus, we obtain

$$
\|Q\|_{2} \geq|r| \sqrt{\frac{q_{n} q_{n-1}}{a}} .
$$

Now, for $|r|<1$, we give an upper bound for the spectral norm of the matrix $Q$. Let the matrices $D$ and $E$ be

$$
D=\left[\begin{array}{ccccc}
\left(\frac{b}{a}\right)^{\frac{\xi(1)}{2}} q_{0} & 1 & 1 & \ldots & 1 \\
r & \left(\frac{b}{a}\right)^{\frac{\xi(1)}{2}} q_{0} & 1 & \ldots & 1 \\
r & r & \left(\frac{b}{a}\right)^{\frac{\xi(1)}{2}} q_{0} & \ldots & 1 \\
\vdots & \vdots & \vdots & \ddots & \vdots \\
r & r & r & \ldots & \left(\frac{b}{a}\right)^{\frac{\xi(1)}{2}} q_{0}
\end{array}\right]
$$

and

$$
E=\left[\begin{array}{ccccc}
\left(\frac{b}{a}\right)^{\frac{\xi(1)}{2}} q_{0} & \left(\frac{b}{a}\right)^{\frac{\xi(2)}{2}} q_{1} & \left(\frac{b}{a}\right)^{\frac{\xi(3)}{2}} q_{2} & \ldots & \left(\frac{b}{a}\right)^{\frac{\xi(n)}{2}} q_{n-1} \\
\left(\frac{b}{a}\right)^{\frac{\xi(n)}{2}} q_{n-1} & \left(\frac{b}{a}\right)^{\frac{\xi(1)}{2}} q_{0} & \left(\frac{b}{a}\right)^{\frac{\xi(2)}{2}} q_{1} & \ldots & \left(\frac{b}{a}\right)^{\frac{\xi(n-1)}{2}} q_{n-2} \\
\left(\frac{b}{a}\right)^{\frac{\xi(n-1)}{2}} q_{n-2} & \left(\frac{b}{a}\right)^{\frac{\xi(n)}{2}} q_{n-1} & \left(\frac{b}{a}\right)^{\frac{\xi(1)}{2}} q_{0} & \ldots & \left(\frac{b}{a}\right)^{\frac{\xi(n-2)}{2}} q_{n-3} \\
\vdots & \vdots & \vdots & \ddots & \vdots \\
\left(\frac{b}{a}\right)^{\frac{\xi(2)}{2}} q_{1} & \left(\frac{b}{a}\right)^{\frac{\xi(3)}{2}} q_{2} & \left(\frac{b}{a}\right)^{\frac{\xi(4)}{2}} q_{3} & \ldots & \left(\frac{b}{a}\right)^{\frac{\xi(1)}{2}} q_{0}
\end{array}\right],
$$


so that $Q=D \circ E$. Then we obtain

$$
\begin{aligned}
& r_{1}(D)=\max _{1 \leq i \leq n} \sqrt{\sum_{j=1}^{n}\left|d_{i j}\right|^{2}}=\sqrt{\left(\frac{b}{a}\right)^{\frac{\xi(1)}{2}} q_{0}^{2}+(n-1)}=\sqrt{n-1} \\
& c_{1}(E)=\max _{1 \leq j \leq n} \sqrt{\sum_{i=1}^{n}\left|e_{i j}\right|^{2}}=\sqrt{\sum_{k=0}^{n-1}\left(\frac{b}{a}\right)^{\xi(k+1)} q_{k}^{2}}=\sqrt{\frac{q_{n} q_{n-1}}{a}} .
\end{aligned}
$$

By Lemma 1.4 we have

$$
\|Q\|_{2} \leq r_{1}(D) c_{1}(E)=\sqrt{(n-1) \frac{q_{n} q_{n-1}}{a}} .
$$

Thus,

$$
|r| \sqrt{\frac{q_{n} q_{n-1}}{a}} \leq\|Q\|_{2} \leq \sqrt{(n-1) \frac{q_{n} q_{n-1}}{a}} .
$$

Theorem 2.3 Let $L=C_{r}\left(\left(\frac{b}{a}\right)^{\frac{\xi(0)}{2}} l_{0},\left(\frac{b}{a}\right)^{\frac{\xi(1)}{2}} l_{1},\left(\frac{b}{a}\right)^{\frac{\xi(2)}{2}} l_{2}, \ldots,\left(\frac{b}{a}\right)^{\frac{\xi(n-1)}{2}} l_{n-1}\right)$ be an r-circulant matrix. Then, for $r \in \mathbb{C}$, we have:

$$
\text { if }|r| \geq 1 \text {, then }
$$

$$
\sqrt{\frac{l_{n} l_{n-1}}{a}+2} \leq\|L\|_{2} \leq|r|\left(\frac{l_{n} l_{n-1}}{a}+2\right)
$$

if $|r|<1$, then

$$
|r| \sqrt{\frac{l_{n} l_{n-1}}{a}+2} \leq\|L\|_{2} \leq \sqrt{n\left(\frac{l_{n} l_{n-1}}{a}+2\right)} .
$$

Proof The matrix $L$ is of the form

$$
L=\left[\begin{array}{ccccc}
\left(\frac{b}{a}\right)^{\frac{\xi(0)}{2}} l_{0} & \left(\frac{b}{a}\right)^{\frac{\xi(1)}{2}} l_{1} & \left(\frac{b}{a}\right)^{\frac{\xi(2)}{2}} l_{2} & \ldots & \left(\frac{b}{a}\right)^{\frac{\xi(n-1)}{2}} l_{n-1} \\
r\left(\frac{b}{a}\right)^{\frac{\xi(n-1)}{2}} l_{n-1} & \left(\frac{b}{a}\right)^{\frac{\xi(0)}{2}} l_{0} & \left(\frac{b}{a}\right)^{\frac{\xi(1)}{2}} l_{1} & \ldots & \left(\frac{b}{a}\right)^{\frac{\xi(n-2)}{2}} l_{n-2} \\
r\left(\frac{b}{a}\right)^{\frac{\xi(n-2)}{2}} l_{n-2} & r\left(\frac{b}{a}\right)^{\frac{\xi(n-1)}{2}} l_{n-1} & \left(\frac{b}{a}\right)^{\frac{\xi(0)}{2}} l_{0} & \ldots & \left(\frac{b}{a}\right)^{\frac{\xi(n-3)}{2}} l_{n-3} \\
\vdots & \vdots & \vdots & \ddots & \vdots \\
r\left(\frac{b}{a}\right)^{\frac{\xi(1)}{2}} l_{1} & r\left(\frac{b}{a}\right)^{\frac{\xi(2)}{2}} l_{2} & r\left(\frac{b}{a}\right)^{\frac{\xi(3)}{2}} l_{3} & \ldots & \left(\frac{b}{a}\right)^{\frac{\xi(0)}{2}} l_{0}
\end{array}\right] .
$$

Then we have

$$
\|L\|_{F}^{2}=\sum_{k=0}^{n-1}(n-k)\left(\frac{b}{a}\right)^{\xi(k)} l_{k}^{2}+\sum_{k=1}^{n-1} k|r|^{2}\left(\frac{b}{a}\right)^{\xi(k)} l_{k}^{2}
$$


Hence, for $|r| \geq 1$, using Eq. (9), we obtain

$$
\begin{aligned}
\|L\|_{F}^{2} & \geq \sum_{k=0}^{n-1}(n-k)\left(\frac{b}{a}\right)^{\xi(k)} l_{k}^{2}+\sum_{k=1}^{n-1} k\left(\frac{b}{a}\right)^{\xi(k)} l_{k}^{2} \\
& =n \sum_{k=0}^{n-1}\left(\frac{b}{a}\right)^{\xi(k)} l_{k}^{2} \\
& =n\left(\frac{l_{n} l_{n-1}}{a}+2\right)
\end{aligned}
$$

that is,

$$
\frac{1}{\sqrt{n}}\|L\|_{F} \geq \sqrt{\frac{l_{n} l_{n-1}}{a}+2 .}
$$

From (6) we have

$$
\|L\|_{2} \geq \sqrt{\frac{l_{n} l_{n-1}}{a}+2} .
$$

Now, for $|r| \geq 1$, we give an upper bound for the spectral norm of the matrix $L$. Let the matrices $F$ and $H$ be

$$
F=\left[\begin{array}{ccccc}
r\left(\frac{b}{a}\right)^{\frac{\xi(0)}{2}} l_{0} & 1 & 1 & \ldots & 1 \\
r\left(\frac{b}{a}\right)^{\frac{\xi(n-1)}{2}} l_{n-1} & r\left(\frac{b}{a}\right)^{\frac{\xi(0)}{2}} l_{0} & 1 & \ldots & 1 \\
r\left(\frac{b}{a}\right)^{\frac{\xi(n-2)}{2}} l_{n-2} & r\left(\frac{b}{a}\right)^{\frac{\xi(n-1)}{2}} l_{n-1} & r\left(\frac{b}{a}\right)^{\frac{\xi(0)}{2}} l_{0} & \ldots & 1 \\
\vdots & \vdots & \vdots & \ddots & \vdots \\
r\left(\frac{b}{a}\right)^{\frac{\xi(1)}{2}} l_{1} & r\left(\frac{b}{a}\right)^{\frac{\xi(2)}{2}} l_{2} & r\left(\frac{b}{a}\right)^{\frac{\xi(3)}{2}} l_{3} & \ldots & r\left(\frac{b}{a}\right)^{\frac{\xi(0)}{2}} l_{0}
\end{array}\right]
$$

and

$$
H=\left[\begin{array}{ccccc}
\left(\frac{b}{a}\right)^{\frac{\xi(0)}{2}} l_{0} & \left(\frac{b}{a}\right)^{\frac{\xi(1)}{2}} l_{1} & \left(\frac{b}{a}\right)^{\frac{\xi(2)}{2}} l_{2} & \ldots & \left(\frac{b}{a}\right)^{\frac{\xi(n-1)}{2}} l_{n-1} \\
1 & \left(\frac{b}{a}\right)^{\frac{\xi(0)}{2}} l_{0} & \left(\frac{b}{a}\right)^{\frac{\xi(1)}{2}} l_{1} & \ldots & \left(\frac{b}{a}\right)^{\frac{\xi(n-2)}{2}} l_{n-2} \\
1 & 1 & \left(\frac{b}{a}\right)^{\frac{\xi(0)}{2}} l_{0} & \ldots & \left(\frac{b}{a}\right)^{\frac{\xi(n-3)}{2}} l_{n-3} \\
\vdots & \vdots & \vdots & \ddots & \vdots \\
1 & 1 & 1 & \ldots & \left(\frac{b}{a}\right)^{\frac{\xi(0)}{2}} l_{0}
\end{array}\right]
$$

so that $L=F \circ H$. Then we obtain

$$
\begin{aligned}
& r_{1}(F)=\max _{1 \leq i \leq n} \sqrt{\sum_{j=1}^{n}\left|f_{i j}\right|^{2}}=\sqrt{|r|^{2} \sum_{k=0}^{n-1}\left(\frac{b}{a}\right)^{\xi(k)}} l_{k}^{2}=|r| \sqrt{\frac{l_{n} l_{n-1}}{a}+2}, \\
& c_{1}(H)=\max _{1 \leq j \leq n} \sqrt{\sum_{i=1}^{n}\left|h_{i j}\right|^{2}}=\sqrt{\sum_{k=0}^{n-1}\left(\frac{b}{a}\right)^{\xi(k)} l_{k}^{2}}=\sqrt{\frac{l_{n} l_{n-1}}{a}+2 .}
\end{aligned}
$$


By Lemma 1.4 we have

$$
\|L\|_{2} \leq r_{1}(F) c_{1}(H)=|r|\left(\frac{l_{n} l_{n-1}}{a}+2\right)
$$

Thus,

$$
\sqrt{\frac{l_{n} l_{n-1}}{a}+2} \leq\|L\|_{2} \leq|r|\left(\frac{l_{n} l_{n-1}}{a}+2\right)
$$

On the other hand, for $|r|<1$, we have

$$
\begin{aligned}
\|L\|_{F}^{2} & \geq \sum_{k=0}^{n-1}(n-k)|r|^{2}\left(\frac{b}{a}\right)^{\xi(k)} l_{k}^{2}+\sum_{k=1}^{n-1} k|r|^{2}\left(\frac{b}{a}\right)^{\xi(k)} l_{k}^{2} \\
& =n|r|^{2} \sum_{k=0}^{n-1}\left(\frac{b}{a}\right)^{\xi(k)} l_{k}^{2} \\
& =n|r|^{2}\left(\frac{l_{n} l_{n-1}}{a}+2\right),
\end{aligned}
$$

that is,

$$
\frac{1}{\sqrt{n}}\|L\|_{F} \geq|r| \sqrt{\frac{l_{n} l_{n-1}}{a}+2} .
$$

Thus, we obtain

$$
\|L\|_{2} \geq|r| \sqrt{\frac{l_{n} l_{n-1}}{a}+2}
$$

Now, for $|r|<1$, we give an upper bound for the spectral norm of the matrix $L$. Let the matrices $G$ and $K$ be

$$
G=\left[\begin{array}{ccccc}
1 & 1 & 1 & \ldots & 1 \\
r & 1 & 1 & \ldots & 1 \\
r & r & 1 & \ldots & 1 \\
\vdots & \vdots & \vdots & \ddots & \vdots \\
r & r & r & \ldots & 1
\end{array}\right]
$$

and

$$
K=\left[\begin{array}{ccccc}
\left(\frac{b}{a}\right)^{\frac{\xi(0)}{2}} l_{0} & \left(\frac{b}{a}\right)^{\frac{\xi(1)}{2}} l_{1} & \left(\frac{b}{a}\right)^{\frac{\xi(2)}{2}} l_{2} & \ldots & \left(\frac{b}{a}\right)^{\frac{\xi(n-1)}{2}} l_{n-1} \\
\left(\frac{b}{a}\right)^{\frac{\xi(n-1)}{2}} l_{n-1} & \left(\frac{b}{a}\right)^{\frac{\xi(0)}{2}} l_{0} & \left(\frac{b}{a}\right)^{\frac{\xi(1)}{2}} l_{1} & \ldots & \left(\frac{b}{a}\right)^{\frac{\xi(n-2)}{2}} l_{n-2} \\
\left(\frac{b}{a}\right)^{\frac{\xi(n-2)}{2}} l_{n-2} & \left(\frac{b}{a}\right)^{\frac{\xi(n-1)}{2}} l_{n-1} & \left(\frac{b}{a}\right)^{\frac{\xi(0)}{2}} l_{0} & \ldots & \left(\frac{b}{a}\right)^{\frac{\xi(n-3)}{2}} l_{n-3} \\
\vdots & \vdots & \vdots & \ddots & \vdots \\
\left(\frac{b}{a}\right)^{\frac{\xi(1)}{2}} l_{1} & \left(\frac{b}{a}\right)^{\frac{\xi(2)}{2}} l_{2} & \left(\frac{b}{a}\right)^{\frac{\xi(3)}{2}} l_{3} & \ldots & \left(\frac{b}{a}\right)^{\frac{\xi(0)}{2}} l_{0}
\end{array}\right],
$$


so that $L=G \circ K$. Then we obtain

$$
\begin{aligned}
& r_{1}(G)=\max _{1 \leq i \leq n} \sqrt{\sum_{j=1}^{n}\left|g_{i j}\right|^{2}}=\sqrt{n}, \\
& c_{1}(K)=\max _{1 \leq j \leq n} \sqrt{\sum_{i=1}^{n}\left|k_{i j}\right|^{2}}=\sqrt{\sum_{k=0}^{n-1}\left(\frac{b}{a}\right)^{\xi(k)} l_{k}^{2}}=\sqrt{\frac{l_{n} l_{n-1}}{a}+2 .}
\end{aligned}
$$

By Lemma 1.4 we have

$$
\|L\|_{2} \leq r_{1}(G) c_{1}(K)=\sqrt{n\left(\frac{l_{n} l_{n-1}}{a}+2\right)} .
$$

Thus,

$$
|r| \sqrt{\frac{l_{n} l_{n-1}}{a}+2} \leq\|L\|_{2} \leq \sqrt{n\left(\frac{l_{n} l_{n-1}}{a}+2\right)} .
$$

Corollary 2.1 Let $Q=C_{r}\left(\left(\frac{b}{a}\right)^{\frac{\xi(1)}{2}} q_{0},\left(\frac{b}{a}\right)^{\frac{\xi(2)}{2}} q_{1},\left(\frac{b}{a}\right)^{\frac{\xi(3)}{2}} q_{2}, \ldots,\left(\frac{b}{a}\right)^{\frac{\xi(n)}{2}} q_{n-1}\right)$ and $\quad L=$ $C_{r}\left(\left(\frac{b}{a}\right)^{\frac{\xi(0)}{2}} l_{0},\left(\frac{b}{a}\right)^{\frac{\xi(1)}{2}} l_{1},\left(\frac{b}{a}\right)^{\frac{\xi(2)}{2}} l_{2}, \ldots,\left(\frac{b}{a}\right)^{\frac{\xi(n-1)}{2}} l_{n-1}\right)$ be r-circulant matrices, where $r \in \mathbb{C}$.

(i) If $|r| \geq 1$, then

$$
\|Q \circ L\|_{2} \leq|r|^{2} \frac{q_{n} q_{n-1}}{a}\left(\frac{l_{n} l_{n-1}}{a}+2\right) .
$$

(ii) If $|r|<1$, then

$$
\|Q \circ L\|_{2} \leq \sqrt{n(n-1) \frac{q_{n} q_{n-1}}{a}\left(\frac{l_{n} l_{n-1}}{a}+2\right)} .
$$

Proof Since $\|Q \circ L\|_{2} \leq\|Q\|_{2}\|L\|_{2}$, the proof is trivial by Theorems 2.2 and 2.3.

Corollary 2.2 Let $Q=C_{r}\left(\left(\frac{b}{a}\right)^{\frac{\xi(1)}{2}} q_{0},\left(\frac{b}{a}\right)^{\frac{\xi(2)}{2}} q_{1},\left(\frac{b}{a}\right)^{\frac{\xi(3)}{2}} q_{2}, \ldots,\left(\frac{b}{a}\right)^{\frac{\xi(n)}{2}} q_{n-1}\right)$ and $L=$ $C_{r}\left(\left(\frac{b}{a}\right)^{\frac{\xi(0)}{2}} l_{0},\left(\frac{b}{a}\right)^{\frac{\xi(1)}{2}} l_{1},\left(\frac{b}{a}\right)^{\frac{\xi(2)}{2}} l_{2}, \ldots,\left(\frac{b}{a}\right)^{\frac{\xi(n-1)}{2}} l_{n-1}\right)$ be r-circulant matrices, where $r \in \mathbb{C}$.

(i) If $|r| \geq 1$, then

$$
\|Q \otimes L\|_{2} \geq \sqrt{\frac{q_{n} q_{n-1}}{a}\left(\frac{l_{n} l_{n-1}}{a}+2\right)}
$$

and

$$
\|Q \otimes L\|_{2} \leq|r|^{2} \frac{q_{n} q_{n-1}}{a}\left(\frac{l_{n} l_{n-1}}{a}+2\right) l .
$$

(ii) If $|r|<1$, then

$$
\|Q \otimes L\|_{2} \geq|r|^{2} \sqrt{\frac{q_{n} q_{n-1}}{a}\left(\frac{l_{n} l_{n-1}}{a}+2\right)}
$$


and

$$
\|Q \otimes L\|_{2} \leq \sqrt{n(n-1) \frac{q_{n} q_{n-1}}{a}\left(\frac{l_{n} l_{n-1}}{a}+2\right)} .
$$

Proof Since $\|Q \otimes L\|_{2}=\|Q\|_{2}\|L\|_{2}$, the proof is trivial by Theorems 2.2 and 2.3.

\section{Conclusion}

In this paper, we obtain new upper and lower bounds for the spectral norms of the $r$ circulant matrices $Q$ and $L$ whose entries are the biperiodic Fibonacci and biperiodic Lucas numbers. This study can be reduced to various studies for the specific values of $a$ and $b$ in the literature. For example, if $a=b=r=1, a=b=1$, and $a=b=k$ in $Q$ and $L$, our results reduce to the studies $[13,18]$, and [19], respectively. Since this study is a generalization of these studies, it contributes to the literature by providing essential information on the spectral norms of $r$-circulant matrices.

\section{Acknowledgements}

The authors are grateful to the anonymous referees who have contributed to improve the quality of the paper. The authors declare that they have not received any financial support for this research.

\section{Competing interests}

The authors declare that there are no competing interests with any individual or institution.

\section{Authors' contributions}

Both authors contributed equally to the writing of this paper. Both authors read and approved the final manuscript.

\section{Publisher's Note}

Springer Nature remains neutral with regard to jurisdictional claims in published maps and institutional affiliations.

Received: 24 April 2017 Accepted: 3 August 2017 Published online: 18 August 2017

\section{References}

1. Koshy, T: Fibonacci and Lucas Numbers with Applications. Wiley, New York (2011)

2. Yazlik, Y, Taskara, N: A note on generalized k-Horadam sequence. Comput. Math. Appl. 63(1), 36-41 (2012)

3. Edson, M, Yayenie, O: A new generalization of Fibonacci sequence \& extended Binet's formula. Integers 9(6), 639-654 (2009)

4. Bilgici, G: Two generalizations of Lucas sequence. Appl. Math. Comput. 245, 526-538 (2014)

5. Yayenie, O: New identities for generalized Fibonacci sequences and new generalization of Lucas sequences. Southeast Asian Bull. Math. 36(5), 739-752 (2012)

6. Falcón, S, Plaza, Á:The k-Fibonacci sequence and the Pascal 2-triangle. Chaos Solitons Fractals 33(1), 38-49 (2007)

7. Falcon, S: On the k-Lucas numbers. Int. J. Contemp. Math. Sci. 6(21), 1039-1050 (2011)

8. Yazlık, Y, Köme, C, Madhusudanan, V: A new generalization of Fibonacci and Lucas p-numbers. J. Comput. Anal. Appl. 25(4), 657-669 (2018)

9. Kocer, EG, Tuglu, N, Stakhov, A: On the m-extension of the Fibonacci and Lucas $p$-numbers. Chaos Solitons Fractals 40(4), 1890-1906 (2009)

10. Kilic, E: The Binet formula, sums and representations of generalized Fibonacci $p$-numbers. Eur. J. Comb. 29(3), 701-711 (2008)

11. Stakhov, A, Rozin, B: Theory of Binet formulas for Fibonacci and Lucas p-numbers. Chaos Solitons Fractals 27(5), 1162-1177 (2006)

12. Uslu, K, Taskara, N, Kose, H: The generalized k-Fibonacci and k-Lucas numbers. Ars Comb. 99, 25-32 (2011)

13. Solak, S: On the norms of circulant matrices with the Fibonacci and Lucas numbers. Appl. Math. Comput. 160(1), 125-132 (2005)

14. Bahsi, M, Solak, S: On the norms of $r$-circulant matrices with the hyper-Fibonacci and Lucas numbers. J. Math. Inequal. 8(4), 693-705 (2014)

15. Bahsi, M: On the norms of circulant matrices with the generalized Fibonacci and Lucas numbers. TWMS J. Pure Appl. Math. 6(1), 84-92 (2015)

16. Bahsi, M: On the norms of $r$-circulant matrices with the hyperharmonic numbers. J. Math. Inequal. 10(2), 445-458 (2016)

17. Kocer, EG, Mansour, T, Tuglu, N: Norms of circulant and semicirculant matrices with Horadam's numbers. Ars Comb. $85,353-359(2007)$

18. Shen, $\mathrm{S}, \mathrm{Cen}, \mathrm{J}$ : On the bounds for the norms of $r$-circulant matrices with the Fibonacci and Lucas numbers. Appl. Math. Comput. 216(10), 2891-2897 (2010) 
19. Shen, $S$, Cen, J: On the spectral norms of $r$-circulant matrices with the $k$-Fibonacci and $k$-Lucas numbers. Int. J. Contemp. Math. Sci. 5(12), 569-578 (2010)

20. Yazlik, Y, Taskara, N: On the norms of an $r$-circulant matrix with the generalized $k$-Horadam numbers. J. Inequal. Appl. 2013(1), Article ID 394 (2013)

21. Yazlik, Y, Taskara, N: Spectral norm, eigenvalues and determinant of circulant matrix involving the generalized k-Horadam numbers. Ars Comb. 104, 505-512 (2012)

22. Yazlik, Y, Taskara, N: On the inverse of circulant matrix via generalized $k$-Horadam numbers. Appl. Math. Comput. 223, 191-196 (2013)

23. Liu, L: On the spectrum and spectral norms of circulant matrices with generalized Horadam numbers entries. Int. J. Comput. Math. 2014, Article ID 795175 (2014)

24. Kızlateş, C, Tuglu, N: On the bounds for the spectral norms of geometric circulant matrices. J. Inequal. Appl. 2016(1), Article ID 312 (2016)

25. He, C, Ma, J, Zhang, K, Wang, Z: The upper bound estimation on the spectral norm of $r$-circulant matrices with the Fibonacci and Lucas numbers. J. Inequal. Appl. 2015(1), Article ID 72 (2015)

26. Radičić, B: On k-circulant matrices (with geometric sequence). Quaest. Math. 39(1), 135-144 (2016)

27. Shen, S-Q, Cen, J-M: On the norms of circulant matrices with the $(k, h)$-Fibonacci and $(k, h)$-Lucas numbers. Int. J. Contemp. Math. Sci. 6(18), 887-894 (2011)

28. Horn, RA, Johnson, CR: Topics in Matrix Analysis. Cambridge University Press, New York (1991)

29. Mathias, R: The spectral norm of a nonnegative matrix. Linear Algebra Appl. 139, 269-284 (1990)

\section{Submit your manuscript to a SpringerOpen ${ }^{\circ}$ journal and benefit from:}

- Convenient online submission

- Rigorous peer review

- Open access: articles freely available online

- High visibility within the field

- Retaining the copyright to your article

Submit your next manuscript at $\gg$ springeropen.com 